\title{
Prevalence of Malaria and Associated Knowledge, Attitude, and Practice among Suspected Patients in Bahir Dar Zuria District, Northwest Ethiopia
}

\author{
Zelalem Dejazmach $\mathbb{D}^{1}{ }^{1}$ Getaneh Alemu $\mathbb{D}^{2}{ }^{2}$ Mulat Yimer, ${ }^{2,3}$ Banchamlak Tegegne, ${ }^{3}$ \\ and Abel Getaneh ${ }^{4}$ \\ ${ }^{1}$ Department of Medical Laboratory Science, College of Health Sciences, Woldia University, Woldia, Ethiopia \\ ${ }^{2}$ Department of Medical Laboratory Science, College of Medicine and Health Sciences, Bahir Dar University, Bahir Dar, Ethiopia \\ ${ }^{3}$ Medical Parasitology, Amhara Public Health Institute, Bahir Dar, Ethiopia \\ ${ }^{4}$ Medical Parasitology and Vector Control, Bahir Dar University, Bahir Dar, Ethiopia
}

Correspondence should be addressed to Zelalem Dejazmach; zelalemdejazmach@gmail.com

Received 11 July 2021; Revised 29 September 2021; Accepted 5 October 2021; Published 19 October 2021

Academic Editor: Antonella Gigantesco

\begin{abstract}
Copyright (C) 2021 Zelalem Dejazmach et al. This is an open access article distributed under the Creative Commons Attribution License, which permits unrestricted use, distribution, and reproduction in any medium, provided the original work is properly cited.
\end{abstract}

\begin{abstract}
Background. Control and prevention activities have brought substantial decline of malaria incidence in the last two decades in Ethiopia. However, lack of local data on the disease transmission and community knowledge, attitude, and practice about malaria are thought to reverse the trend of malaria in certain areas. Therefore, assessment of the prevalence and community awareness towards malaria plays pivotal role for the success of malaria control and prevention. Objective. To assess malaria prevalence and knowledge, attitude, and practice about malaria among febrile patients in Bahir Dar Zuria district, Northwest Ethiopia. Methods. A facility based crosssectional study was conducted from January to March 2020 among 149 febrile patients attending selected health centers in Bahir Dar Zuria district. Data about knowledge, attitude, and practice about malaria were collected using semistructured questionnaire. Blood sample from each participant was tested for Plasmodium species through malaria rapid diagnostic tests and blood film microscopy. Data were analyzed using statistical software for social sciences version 20. Results. Among 149 participants, 22 (14.8\%) were positive for Plasmodium infection at least by one diagnostic methods. Prevalence of $P$. falciparum and P. vivax was $3.4 \%$ and $10.1 \%$, respectively, while that of mixed infection was $1.3 \%$. From the total study participants, $29.5 \%$ have good knowledge, $77.2 \%$ have positive attitude, and $34.9 \%$ have good practice towards malaria. Statistically significant associations were observed on knowledge with age group $\left(X^{2}=10.377, P=0.035\right)$, educational level $\left(X^{2}=15.075, P=0.001\right)$, family size $\left(X^{2}=7.601, P=0.022\right)$, attitude level and practice level. Participants with family size $<5$ were 6.841 (95\% CI: $2.570-18.206, P \leq 0.001)$ times more likely to have negative attitude as compared to those with family size $\geq 5$. Conclusions. Prevalence of malaria in the study area was relatively high. Study participants had encouraging attitude; however, their knowledge and practice towards malaria were poor. Therefore, the existing malaria control activities should be supplemented with continuous health educations, aware the community, and ensure participation in the control and prevention activities.
\end{abstract}

\section{Background}

Malaria is a febrile disease caused by protozoan parasites of the genus Plasmodium. The disease remains one of the most severe public health problem worldwide, particularly in tropical and subtropical areas [1]. Plasmodium $(P)$ falciparum, $P$. vivax, $P$. malarae, $P$. ovale, and $P$. knowlesi naturally infect human to cause malaria. However, $P$. falciparum and $P$. vivax are the most prevalent malaria parasites globally, the former being the most pathogenic [2].

In 2018, an estimated 228 million cases of malaria occurred leading to a death toll of 405, 000 worldwide [2]. 
Malaria remains to be the major public health problem in Ethiopia [3]. It is estimated that $\sim 68 \%$ of the population is at risk of the disease and a point estimate of 2,362,979 cases and 4,757 deaths were reported in 2018 in the country [2]. Plasmodium falciparum and $P$. vivax are the two dominant species in Ethiopia which accounts $60 \%$ and $40 \%$ of malaria cases, respectively, and are prevalent in all malaria endemic areas. However, the proportion varies among geographical settings and season. In lowlands and during the major malaria transmission season, $P$. falciparum is much common while $P$. vivax is more common at higher altitudes and during the dry season [4]. Malaria is mainly seasonal, and transmission primarily occurs at altitudes below 2,000 meters above sea level in the country [5].

The disease is mainly transmitted through the bite of a female Anopheles mosquito [1], and the clinical symptoms are caused by asexual blood stages of the parasite [6]. The first signs and symptoms of malaria are nonspecific presenting with headache, fatigue, and abdominal discomfort followed by fever, perspiration, chills, and rigors. If left untreated, severe malaria develops which comprises of impaired consciousness, respiratory distress, metabolic acidosis, hypoglycemia, acute kidney injury, circulatory collapse, and pulmonary oedema [7]. Most severe and complicated manifestations and almost all malaria deaths are caused by $P$. falciparum [5].

Malaria-suspected patients should be confirmed by laboratory diagnosis and treated with efficacious antimalaria drugs within 24 hours of the onset of symptoms [8]. Artemisinin combination therapy and chloroquine are the first line drugs for the treatment of uncomplicated $P$. falciparum and $P$. vivax malaria, respectively, and intravenous artesunate or quinine therapy for complicated malaria [5]. Early diagnosis and immediate treatment is one of the main strategies in malaria prevention, control, and effective case management [8]. Moreover, there are also intervention strategies for malaria control activities that have brought substantial reduction of malaria incidence including environmental management, indoor residual spraying (IRS), and use of long-lasting insecticide-treated nets (ITNs) [1].

An updated data in the local prevalence of malaria helps not only to evaluate the local implementation of control and prevention activities but also to aware the healthcare providers and the community as well. On the other hand, many of the recently published epidemiological studies focus on asymptomatic participants by considering their role as potential sources of infection. However, data about the proportion of malaria patients among febrile cases helps to figure out the morbidity impact of the disease and for future planning.

Similarly, understanding knowledge level, perception, and practical behaviors of communities towards malaria is crucial in order to ensure appropriate intervention measures [9]. Different reports regarding the knowledge on malaria in different parts of Africa and around the world documented that there is a knowledge, attitude, and practice (KAP) gap among communities hindering people not to engage actively in the existing intervention programs [10]. People who have good KAP are believed to be in a better position to be protected against malaria $[11,12]$. Findings of this study make inroads into the implementation of effective interventions in the area and indicate focus areas for enhancing community awareness and scaling up coverage of evidence-based interventions. Therefore, this study was conducted to assess prevalence of malaria and KAP among febrile patients in Bahir Dar Zuria district, Northwestern Ethiopia.

\section{Methods}

2.1. Study Design, Area, and Period. A facility-based crosssectional study was conducted in Yinesa, Andasa, and Robit health centers in Bahir Dar Zuria district, Northwest Ethiopia, from January to March 2020. The Bahir Dar Zuria district is one of the 14 districts of West Gojjam Zone, which is located at a distance of $560 \mathrm{~km}$ from capital city of the country, Addis Ababa, and the district is situated surrounding Bahir Dar city, capital of Amhara National Regional State. The altitude of the district ranges from 1700 to 2300 meters above sea level. The district receives an average annual rainfall of about $1035 \mathrm{~mm}$. The minimum and maximum temperature lies at $10^{\circ} \mathrm{C}$ and $32^{\circ} \mathrm{C}$, respectively [13]. The whole land mass of the district is classified as with the major and minor transmission seasons being September to December and April to May, respectively [14]. In the district, there are 9 health centers and 36 health posts. Both health centers and health posts provide diagnostic and treatment services to the community.

2.2. Sample Size Determination and Sampling Technique. The sample size was calculated using single population proportion formula based on the $95 \%$ confidence limits $(\mathrm{Z} \alpha / 2=1.96)$ and $5 \%$ margin of error $(d)$ and previous prevalence $(p)$ of $12.8 \%$ from Shiraro, North Ethiopia [15]. Sample size $=(\mathrm{Z} \alpha / 2)^{2} p(1-p) / d^{2}=(1.96)^{2}(0.128)(1-$ $0.128) /(0.05)^{2}=172$.

Among 9 health centers in the district, 3 were selected for data collection by simple random sampling technique. Accordingly, Andasa, Yinesa, and Robit health centers were selected. Number of study participants from each health center was allocated based on febrile patients flow during the same season of the previous year. Accordingly 50, 60, and 39 febrile patients were included from Andasa, Yinesa, and Robit health centers, respectively. Systematic random sampling was applied to select study participants from each health center. Clinically malaria suspected patients with age $\geq 15$ who were sent to the laboratory for blood film examination and gave consent to participate in the study were included. Critically ill patients who were unable to respond to research questions and individuals who had taken antimalaria drugs within 4 weeks prior to data collection were excluded.

\subsection{Data Collection}

2.3.1. Questionnaire Data. Amharic version of a semistructured questionnaire adapted from recent publications was used to collect data about the sociodemographic characteristics, knowledge, attitude, and practice of respondents related to malaria transmission, symptoms, and prevention 
methods. Questionnaire data were administered through face to face interview. Knowledge assessment questions were asked to each participant, and each correct response was given a score of 1 while a wrong or unsure response was scored 0. Total scores were interpreted based on Bloom's cut-off points $(80.0-100.0 \%$ of correct responses meant a good knowledge, a score of $60.0-79.0 \%$ put a scorer in a level of satisfactory knowledge, and a poor knowledge was for the respondents with a score $\leq 59.0 \%$ of the correct responses were adapted and modified) [16]. Therefore, the scores with their respective knowledge levels were 5.6-7 good knowledge, 4.2-5.5 satisfactory knowledge, and $<4.2$ poor knowledge. Attitude was assessed by Likert's scale [17]. The questions on Likert's scale had positive and negative responses that ranged from agree (score 3 ), undecided (score 2 ), and disagree (score 1). The responses were summed up, and a total score was obtained for each respondent. The mean score was calculated and respondents with score of greater than or equal to the mean score (2.78) were considered as having positive attitude while those with score less than the mean score were taken as having negative attitude towards malaria. Practices were also determined using Likert's type. The scoring system of Likert's type scales with respect to respondents response ranging from never (score 0 ), sometimes (score 1), and always (score 2) were used and interpreted as good and poor practice based on the mean score [18].

2.3.2. Blood Collection and Processing. Capillary blood sample was collected from each participant by finger puncture following standard procedure [5]. After removing the first drop, samples were used for thin and thick blood film preparation as well as for running malaria rapid diagnostic tests (RDTs). A multispecies care start RDT (Access Bio, Belgium) was used in the present study. Thick and thin blood smears for each participant were prepared on the same slide and labeled with sample number and date and let air dried. Then, thin blood smears were fixed with absolute methanol and allowed to air dry. Blood smears were stained with $10 \%$ Giemsa solution and examined with light microscope and reported following the standard protocols [5]. Identification code for each RDT device was given similar to the code used on the slide for each study participant. Then, $5 \mu \mathrm{l}$ of whole blood was added onto the test device window using the specimen transfer device (micropipette) provided with the kit, followed by adding 2 drops $(60 \mu \mathrm{l})$ of reagent buffer, and the result was recorded as per the instruction of the manufacturer.

2.4. Statistical Analysis. Data were checked for completeness and entered and analyzed in statistical package for social sciences (SPSS) version 20. Descriptive statistics was run to measure frequencies and percentages of the variables. Frequency distribution tables were used to quantify sociodemographic variables, knowledge, and attitude of respondents related to symptoms, causes, transmission, prevention, and control measures of malaria as well as practices toward malaria prevention and control methods. Associations between KAP and malaria infection were assessed through logistic regression. Factors associated with KAP level were also analyzed using odds ratio and chi-square. Variables with $P$ value of $<0.3$ in the bivariable analysis were included in the multivariate logistic regression analysis. Variables with $P$ value $<0.05$ in the multivariable analysis were considered as statistically significant at $95 \%$ confidence level.

2.5. Ethics Approval and Consent to Participate. The present research was carried out after ethical approval was obtained from the Institutional Review Committee of College of Medicine and Health Sciences, Bahir Dar University with reference number CMHSc009/2020 on 03 January 2020. Additionally, supportive letters were obtained from Amhara Public Health Institute, West Gojjam Zone Health Department, and Bahir Dar Zuria district health office, and permission was obtained from each health center authorities. Informed verbal consent was obtained from each participant. Consent for participants with age 15-17 was taken in the presence of their parents/guardians. Verbal informed consent was acceptable and approved by the Bahir Dar University, College of Medicine and Health Sciences Research and Ethical Review Committee. Data on decisions of participants to agree or disagree was listed with an excel sheet by their code, and no personal identifier was included as participants were given a unique code. Malaria-suspected patients who were positive for malaria by any of the tests were linked to the respective health center for appropriate treatment.

\section{Results}

3.1. Sociodemographic Characteristics of Study Participants. We were unable to collect data from 172, as planned in the sample size calculation section, due to unprecedented COVID-19 outbreak at the time of data collection. Hence, data from 149 febrile patients was collected and included in the analysis. Among a total of 149 febrile patients participated in the present study, $89(59.7 \%)$ and $60(40.3 \%)$ were males and females, respectively. The participants' age ranged from 15 to 82 years old with mean age of $32.79( \pm 12.91 \mathrm{SD})$ years. All the study participants were rural inhabitants. Regarding to their educational status, $96(64.4 \%)$ were unable to read while write, and the rest 53 (35.6\%) were able to read and write or have attended formal education. More than half (53.7\%) of malaria-suspected patients have history of fever within previous 1 year (Table 1).

3.2. Prevalence of Plasmodium Species. Among 149 participants, $20(13.4 \%)$ and $19(12.8 \%)$ were positive for Plasmodium infection as confirmed by RDTs and microscopy, respectively. Overall, $22(14.8 \%)$ participants were positive by at least either of the diagnostic methods. Species level analysis revealed that the prevalence of $P$. falciparum and P. vivax was $3.4 \%$ and $10.1 \%$, respectively, while that of mixed infection was $1.3 \%$.

3.3. Knowledge of Respondents on Malaria. The study indicated that $144(82.1 \%)$ respondents had heard about malaria. Nearly half of the respondents (48.3\%) attributed the cause of malaria to mosquito bite and the remaining $(49.7 \%)$ did not know the cause of malaria. More than half of 
TABLE 1: Sociodemographic characteristics and clinical data of febrile patients in Bahir Dar Zuria district, Northwest Ethiopia, from January to March 2020.

\begin{tabular}{|c|c|c|c|}
\hline Characteristics & Categories & Frequency & Percentage \\
\hline \multirow{2}{*}{ Sex } & Male & 89 & 59.7 \\
\hline & Female & 60 & 40.3 \\
\hline \multirow{4}{*}{ Age group (in years) } & $15-25$ & 55 & 36.9 \\
\hline & $26-35$ & 36 & 24.2 \\
\hline & $36-45$ & 38 & 25.5 \\
\hline & $\geq 46$ & 20 & 13.4 \\
\hline \multirow{4}{*}{ Educational level } & Unable to read and write & 96 & 64.4 \\
\hline & Able to read and write & 12 & 8.1 \\
\hline & Primary & 24 & 16.1 \\
\hline & Secondary and above & 17 & 11.4 \\
\hline \multirow{2}{*}{ Primary occupation } & Farmer & 124 & 83.2 \\
\hline & Nonfarmer & 25 & 16.8 \\
\hline \multirow{2}{*}{ Marital status } & Single & 30 & 20.1 \\
\hline & Married & 119 & 79.1 \\
\hline \multirow{2}{*}{ Family size } & $<5$ & 75 & 50.3 \\
\hline & $\geq 5$ & 74 & 49.7 \\
\hline \multirow{2}{*}{ History of fever within previous 1 year } & Yes & 69 & 46.3 \\
\hline & No & 80 & 53.7 \\
\hline \multirow{3}{*}{ Causes of previous fever } & Malaria & 26 & 37.7 \\
\hline & Other & 17 & 24.6 \\
\hline & Do not know & 26 & 37.7 \\
\hline
\end{tabular}

respondents identified the major sign and symptoms of malaria correctly; 83 (55.7\%), 83 (55.7\%), and $94(63.1 \%)$ mentioned fever, headache, and chills/shivering as symptoms of malaria, respectively. Fifty (33.6\%) and 26 (17.4\%) participants identified correctly under five children and pregnant women as the most susceptible segments of the population to malaria, respectively. In response to knowledge about prevention strategies, majority of the respondents (81.9\%) mentioned that ITN as malaria preventive method while only 6 (4.0\%) mentioned that malaria can be prevented using IRS. Only 44 (29.5\%) participants responded that avoiding stagnant water prevents from malaria (Table 2).

Pearson's chi-square analyses revealed that age group $\left(x^{2}=10.377, P=0.035\right)$ was significantly associated with participants' knowledge that proportion of good knowledge was highest among $\geq 36$-year-old patients. Similarly, educational status, family size, attitude level, and practice level were also associated with participants' knowledge about malaria (Table 3).

3.4. Attitude towards Malaria and Health Seeking Behavior. From the total respondents, $130(87.2 \%)$ agreed on seriousness and threat posed by malaria. The majority $(88.6 \%)$ of respondents thought that malaria is curable disease. One hundred twenty-three (82.6\%) participants agreed with the statement that malaria is preventable disease (Table 4 ). The majority $(77.2 \%)$ of study participants had positive attitude while $34(22.8 \%)$ had negative attitude towards malaria.
Statistically significant associations were observed between attitude level and family size that participants with family size $<5$ were 6.841 (95\% CI: $2.570-18.206, P \leq 0.001$ ) times more likely to have negative attitude as compared to those with family size $\geq 5$. All other perceived factors were not associated with attitude level (Table 5).

3.5. Practices of Respondents towards Malaria Control and Prevention. Use of mosquito net was the most frequently applied method for malaria prevention by 117 (78.5\%) respondents. Thirty-six (24.3\%) respondents practiced compound/house sanitation as malaria preventive methods, and $25(16.8 \%)$ did not practice for malaria prevention. The types of roof of almost all the respondents (98.0\%) were corrugated iron and most $(95.3 \%)$ of them owned ITNs (Table 6).

Statistically significant associations were observed between practice level and sex of participants. Males had around $52 \%$ less good practice as compared to females (Table 7).

\section{Discussion}

The overall malaria prevalence in the present study $(14.8 \%)$ was in line with previous findings of $16.0 \%$ from Dilla Town, Southern Ethiopia [19], and results reported in northwestern Tigray, Ethiopia (13.4\%) [15]. However, malaria prevalence in the present study was higher than the study 
TABLE 2: Knowledge of respondents regarding to cause, sign and symptoms, transmission, and preventive methods of malaria, Bahir Dar Zuria district, Northwest Ethiopia, 2020.

\begin{tabular}{|c|c|c|c|}
\hline Variables & Category & Frequency $(N=149)$ & Percentage \\
\hline \multirow{2}{*}{ Do you know malaria } & Yes & 144 & 96.6 \\
\hline & No & 5 & 3.4 \\
\hline \multirow{4}{*}{ Causes of malaria } & Mosquito bite & 72 & 48.3 \\
\hline & Cold temperature & 2 & 1.3 \\
\hline & Poor sanitation & 1 & 0.7 \\
\hline & Unknown & 74 & 49.7 \\
\hline \multirow{5}{*}{ Main malaria transmission seasons* } & Before main rainy season & 9 & 6.0 \\
\hline & Main rainy season & 49 & 32.9 \\
\hline & After main rainy season & 82 & 55.0 \\
\hline & All year round & 12 & 8.1 \\
\hline & Unknown & 6 & 4.0 \\
\hline \multirow{7}{*}{ The main sign and symptoms of malaria* } & Fever/sweat & 83 & 55.7 \\
\hline & Headache & 83 & 55.7 \\
\hline & Feeling cold & 94 & 63.1 \\
\hline & Chills/shivers & 94 & 63.1 \\
\hline & Nausea/vomiting & 20 & 13.4 \\
\hline & Weakness, joint/muscle pain & 38 & 25.5 \\
\hline & Loss of appetite & 46 & 30.9 \\
\hline \multirow{7}{*}{ How do people get malaria?* } & Mosquitoes bite & 72 & 48.3 \\
\hline & Drinking dirty water & 52 & 34.9 \\
\hline & Working in sun & 10 & 6.7 \\
\hline & Did not get enough food & 16 & 10.7 \\
\hline & Leave near collected water & 33 & 22.1 \\
\hline & Working in cold temperature & 20 & 13.4 \\
\hline & I do not know & 35 & 23.5 \\
\hline \multirow{6}{*}{ Which group of people is more affected by malaria?* } & All adult (age $>15)$ & 3 & 2.0 \\
\hline & Children ( $5-15$ years old) & 2 & 1.3 \\
\hline & Children $<5$ years old & 50 & 33.6 \\
\hline & Pregnant women & 26 & 17.4 \\
\hline & All are equally affected & 78 & 52.3 \\
\hline & I do not know & 8 & 5.4 \\
\hline \multirow{5}{*}{ What are the prevention methods of malaria? ${ }^{*}$} & Using bed net & 120 & 81.9 \\
\hline & Draining stagnant water & 44 & 29.5 \\
\hline & Spraying house with chemicals & 6 & 4.0 \\
\hline & Sanitation & 2 & 1.3 \\
\hline & Unknown & 25 & 16.8 \\
\hline
\end{tabular}

${ }^{*}$ More than one answer is possible.

results from Shewa Robit, Ethiopia, (2.8\%) [20]. The variation might be due to study design that the current study was facility based but the study conducted at Shewa Robit was community based. On the other hand, malaria prevalence in the present study was lower as compared to findings of $40.9 \%$ in Kola Diba [21], 25.0\% in East Shewa [22] and 49.4\% in Haro Limmu Woreda [23] all in Ethiopia. The low prevalence could be partly explained by the fact that the current data was collected during the dry (low malaria transmission) season while the previous studies were col- lected during the peak malaria transmission season in the country. Moreover, the difference might be due to variation in malaria endemicity. Another possible reason might be due to the effectiveness of malaria prevention and control strategies in Ethiopia. For example, the study conducted at Kola Diba was almost before 9 years but there have been many control and prevention strategies applied in the country such as increased bed net distribution and IRS.

In the current study, majority (96.6\%) of the respondents had heard about malaria, which was comparable with 
TABLE 3: Crosstabulation of chi-square analysis of association of knowledge with sociodemography, among malaria-suspected cases in Bahir Dar Zuria district, Northwest Ethiopia, 2020.

\begin{tabular}{|c|c|c|c|c|c|c|}
\hline Characteristics & Categories & Poor (\%) & $\begin{array}{c}\text { Knowledge level } \\
\text { Satisfied (\%) }\end{array}$ & Good (\%) & $X^{2}$ & $P$ value \\
\hline \multirow{2}{*}{ Sex } & Male & $32(58.2)$ & $29(58)$ & $28(63.6)$ & \multirow{2}{*}{0.820} & \multirow{2}{*}{0.396} \\
\hline & Female & $23(41.8)$ & $21(42)$ & $16(36.4)$ & & \\
\hline \multirow{3}{*}{ Age group (in years) } & $15-25$ & $29(52.7)$ & $14(28)$ & $12(27.3)$ & \multirow{3}{*}{10.377} & \multirow{3}{*}{0.035} \\
\hline & $26-35$ & $9(16.4)$ & $16(32)$ & $11(25)$ & & \\
\hline & $\geq 36$ & $17(30.9)$ & $20(40)$ & $21(47.7)$ & & \\
\hline \multirow{2}{*}{ Educational level } & Unable to read and write & $41(74.5)$ & $37(74)$ & $18(40.9)$ & \multirow{2}{*}{15.075} & \multirow{2}{*}{0.001} \\
\hline & Able to read and write and above & $14(25.5)$ & $13(26)$ & $26(59.1)$ & & \\
\hline \multirow{2}{*}{ Primary occupation } & Farmer & $47(85.5)$ & $45(90)$ & $32(72.7)$ & \multirow{2}{*}{5.312} & \multirow{2}{*}{0.07} \\
\hline & Nonfarmer & $8(14.5)$ & $5(10)$ & $12(27.3)$ & & \\
\hline \multirow{2}{*}{ Marital status } & Single & $13(23.6)$ & $7(14)$ & $10(22.7)$ & \multirow{2}{*}{1.774} & \multirow{2}{*}{0.412} \\
\hline & Married & $42(76.4)$ & $43(86)$ & $34(77.3)$ & & \\
\hline \multirow{2}{*}{ Family size } & $<5$ & $34(61.8)$ & $26(52)$ & $15(34.1)$ & \multirow{2}{*}{7.601} & \multirow{2}{*}{0.022} \\
\hline & $\geq 5$ & $21(38.2)$ & $24(48)$ & $29(65.9)$ & & \\
\hline
\end{tabular}

TABle 4: Attitude towards malaria and health seeking behavior and information access of the respondents in Bahir Dar Zuria district, Northwest Ethiopia, 2020.

\begin{tabular}{|c|c|c|c|}
\hline Variables & Category & Frequency $(N=149)$ & Percentage \\
\hline \multirow{3}{*}{ Do you think that malaria is public health problem? } & Yes & 130 & 87.2 \\
\hline & No & 10 & 6.7 \\
\hline & I do not know & 9 & 6.0 \\
\hline \multirow{3}{*}{ Do you think that malaria is curable? } & Yes & 132 & 88.6 \\
\hline & No & 12 & 8.1 \\
\hline & I do not know & 5 & 3.4 \\
\hline \multirow{3}{*}{ Is malaria preventable? } & Yes & 123 & 82.6 \\
\hline & No & 16 & 10.7 \\
\hline & I do not know & 10 & 6.7 \\
\hline \multirow{2}{*}{ Duration of the present fever/illness (in hours) } & $\leq 24$ & 41 & 38.9 \\
\hline & $>24$ & 108 & 61.1 \\
\hline \multirow{4}{*}{ Reasons for delay after fever started } & Too far & 8 & 7.4 \\
\hline & Cannot afford cost of transport & 6 & 5.6 \\
\hline & I go if got sever & 84 & 77.8 \\
\hline & Health facility was closed & 10 & 9.3 \\
\hline \multirow{5}{*}{ What did you do first when this fever started } & Went to health facility & 11 & 7.4 \\
\hline & Use herb/natural remedies & 9 & 6 \\
\hline & Went to religious leaders & 8 & 5.4 \\
\hline & Went drug store to get drug & 5 & 3.4 \\
\hline & We have done nothing & 116 & 77.9 \\
\hline \multirow{2}{*}{ Sought treatment before visiting the health center } & Yes & 12 & 8.1 \\
\hline & No & 137 & 91.9 \\
\hline \multirow{2}{*}{ Health education on malaria in the last 6 months } & Yes & 11 & 7.4 \\
\hline & No & 138 & 92.6 \\
\hline
\end{tabular}


TABle 5: Multivariate logistic regression analysis of attitude level among febrile patients in Bahir Dar Zuria district, Northwest Ethiopia, 2020.

\begin{tabular}{|c|c|c|c|c|c|c|c|}
\hline \multirow{2}{*}{ Characteristics } & \multirow{2}{*}{ Categories } & \multicolumn{2}{|c|}{ Attitude level } & \multirow{2}{*}{ COR $(95 \%$ CI $)$} & \multirow{2}{*}{$P$ value } & \multirow{2}{*}{ AOR 95\% CI } & \multirow{2}{*}{$P$ value } \\
\hline & & Negative (\%) & Positive (\%) & & & & \\
\hline \multirow{2}{*}{ Sex } & Male & $22(64.7)$ & $67(58.3)$ & $1.313(0.593-2.909$ & 0.502 & $0.458(0.184-1.136)$ & 0.092 \\
\hline & Female & $12(35.3)$ & $48(41.3)$ & 1 & & & \\
\hline \multirow{3}{*}{ Age group (in years) } & $15-25$ & $17(50)$ & $38(33.0)$ & $0.583(0.248-1.371)$ & 0.216 & $0.851(0.315-2.300)$ & 0.750 \\
\hline & $26-35$ & $5(14.7)$ & $31(27.0)$ & $1.617(0.518-5.049)$ & 0.408 & $2.244(0.660-7.629)$ & 0.196 \\
\hline & $\geq 36$ & $12(35.3)$ & $46(40.0)$ & 1 & & & \\
\hline \multirow[b]{2}{*}{ Educational level } & Unable to read and write & $23(67.6)$ & $73(63.5)$ & $1.203(0.534-2.711)$ & 0.656 & & \\
\hline & $\begin{array}{l}\text { Able to read and write } \\
\text { and above }\end{array}$ & $11(32.4)$ & $42(36.5)$ & 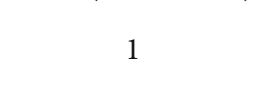 & & & \\
\hline \multirow{2}{*}{ Primary occupation } & Farmer & $26(76.5)$ & $98(85.2)$ & $1.774(0.689-4.564)$ & 0.235 & $1.514(0.503-4.562)$ & 0.461 \\
\hline & Nonfarmer & $8(23.5)$ & $17(14.8)$ & 1 & & & \\
\hline \multirow{2}{*}{ Marital status } & Single & $7(20.6)$ & $23(20.0)$ & $0.964(0.373-2.490)$ & 0.940 & & \\
\hline & Married & $27(79.4)$ & $92(80.0)$ & 1 & & & \\
\hline \multirow{2}{*}{ Family size } & $<5$ & $27(79.4)$ & $48(41.7)$ & $5.384(2.167-13.378)$ & $\leq 0.001$ & $6.841(2.570-18.206)$ & $\leq 0.001$ \\
\hline & $\geq 5$ & $7(20.6)$ & $67(58.3)$ & 1 & & & \\
\hline
\end{tabular}

TABle 6: Practices of respondents towards malaria prevention and control strategies in Bahir Dar Zuria district, northwest Ethiopia, 2020.

\begin{tabular}{|c|c|c|c|}
\hline Variables & Category & Frequency $(N=149)$ & Percentage \\
\hline \multirow{5}{*}{ What do you do in your household to prevent malaria?* } & Using mosquito nets & 117 & 78.5 \\
\hline & Spray house with DDT & 5 & 3.4 \\
\hline & Compound/house sanitation & 36 & 24.2 \\
\hline & Close door and window early night & 6 & 4.0 \\
\hline & Nothing & 25 & 16.8 \\
\hline \multirow{2}{*}{ Type of roof } & Thatched & 3 & 2.0 \\
\hline & Corrugated iron & 146 & 98.0 \\
\hline \multirow{2}{*}{ Household ownership of ITNs } & Yes & 142 & 95.3 \\
\hline & No & 7 & 4.7 \\
\hline \multirow{4}{*}{ Number of ITNs owned } & 1 & 11 & 7.7 \\
\hline & 2 & 35 & 24.6 \\
\hline & 3 & 58 & 40.8 \\
\hline & $\geq 4$ & 38 & 26.8 \\
\hline \multirow{4}{*}{ Frequency of night slept under ITNs in the last 15 days } & All nights & 52 & 36.6 \\
\hline & Sometimes & 13 & 9.2 \\
\hline & Only few nights & 15 & 10.6 \\
\hline & None of the nights & 62 & 43.7 \\
\hline
\end{tabular}

previous study results of $97.6 \%-100.0 \%$ in Ethiopia [20, 24, 25]. However, it was higher than results in Kersa (85.9\%) [26]. Seventy two $(48.3 \%)$ participants implicated mosquito bite was the way of malaria transmission, similarly with results of $47.5 \%$ and $48.8 \%$ o from Assosa [9] and Tigray [27], respectively. On the other hand, it was lower than findings from Raya Azebo district (63\%) [24], Shewa Robit (85.2\%) [20], Tepi Town (86.7\%) [25], Southern Ethiopia (83.7\%) [28], Oromia Region (63.4\%) [29], and Arba Minch $(98.2 \%)$ [30]. It was also lower than findings from Nigeria
(74.3\%) [31], Tanzania (95.31\%) [32], Iran (77.8\%) [33], and Swaziland (92.8\%) [34]. The difference might be due to variation of respondent characteristics like the educational level. In the present study, $64.4 \%$ of the respondents were illiterate, unlike those studies in Ethiopia (5.6\%) [25] and Iran (37.3\%) [33]. Sources of information might be the other reason for malaria transmission awareness difference, as about $92.6 \%$ of the respondents have no source of information in the last six months for malaria in our study. However, this study participants' knowledge about the means of 
TABle 7: Multivariate logistic regression analysis of practice level among malaria-suspected cases in Bahir Dar Zuria district, Northwest Ethiopia, 2020.

\begin{tabular}{|c|c|c|c|c|c|c|c|}
\hline \multirow{2}{*}{ Characteristics } & \multirow{2}{*}{ Categories } & \multicolumn{2}{|c|}{ Practice level } & \multirow{2}{*}{ COR $(95 \% \mathrm{CI})$} & \multirow{2}{*}{$P$ value } & \multirow{2}{*}{ COR $(95 \% \mathrm{CI})$} & \multirow{2}{*}{$P$ value } \\
\hline & & Poor $(\%)$ & Good (\%) & & & & \\
\hline \multirow{2}{*}{ Sex } & Male & $64(71.9)$ & $25(28.1)$ & $0.477(0.240-0.949)$ & 0.035 & $0.476(0.231-0.980)$ & 0.044 \\
\hline & Female & $33(55.0)$ & $27(45.0)$ & 1 & & & \\
\hline \multirow{3}{*}{ Age group (in years) } & $15-25$ & $41(74.5)$ & $14(25.5)$ & $0.484(0.217-1.077)$ & 0.075 & $0.531(0.201-1.398)$ & 0.200 \\
\hline & $26-35$ & $22(61.1)$ & $14(38.9)$ & $0.902(0.385-2.109)$ & 0.811 & $0.838(0.348-2.015)$ & 0.693 \\
\hline & $\geq 36$ & $34(58.6)$ & $24(41.4)$ & 1 & & & \\
\hline \multirow{2}{*}{ Educational level } & Unable to read and write & $61(63.5)$ & $35(36.5)$ & $1.215(0.597-2.474)$ & 0.591 & & \\
\hline & Able to read and write and above & $36(67.9)$ & $17(32.1)$ & 1 & & & \\
\hline \multirow{2}{*}{ Primary occupation } & Farmer & $80(64.5)$ & $44(35.5)$ & $1.169(0.467-2.925)$ & 0.739 & & \\
\hline & Nonfarmer & $17(68.0)$ & $8(32.0)$ & 1 & & & \\
\hline \multirow{2}{*}{ Marital status } & Single & 24() & $6(11.5)$ & $0.397(0.151-1.044)$ & 0.061 & $0.667(0.210-2.182)$ & 0.514 \\
\hline & Married & $73(75.3)$ & $46(88.5)$ & 1 & & & \\
\hline \multirow{2}{*}{ Family size } & $<5$ & $48(49.5)$ & $27(51.9)$ & $1.102(0.562-2.163)$ & 0.777 & & \\
\hline & $\geq 5$ & $49(50.5)$ & $25(48.1)$ & 1 & & & \\
\hline
\end{tabular}

transmission of malaria is much better than other studies conducted in Amhara region where 32.3\% mentioned mosquito bite for transmission of malaria [35]. This variation might also be due to differences in educational level since $67.9 \%$ of the respondents in the previous study were illiterate.

More than half (55.0\%) of the participants responded that main malaria transmission season was after main rainy season. Moreover, 49 (32.9\%) responded during main rainy season and $12(8.1 \%)$ responded all year round as the main malaria transmission season. Similar study conducted in Southern Ethiopia reported that respective proportion of participants responded that main malaria transmission season were cloudy weather $(30.8 \%)$, rainy season $(27.7 \%)$, dry season (32.7\%), and any season (8.8\%) [28].

Most of the respondents $(91.3 \%)$ were familiar with at least one of the classical symptoms of malaria. More than half of respondents identified sign and symptoms of malaria correctly that 83 (55.7\%), 83 (55.7\%), and 94 (63.1\%) mentioned fever, headache, and chills and shivering, respectively. Similar results were found from different KAP studies including Raya Azebo district [24] and Tigray region [27]. However, it was lower than findings from Shewa Robit where $94.4 \%, 84.5 \%$, and $93.3 \%$ mentioned fever, headache, and chills and shivering, respectively [20]. Knowledge regarding malaria signs and symptoms was also lower than other reports in Ethiopia [9, 30] and Karachi [36]. On the contrary, it was higher than study results in Central Africa Republic that respondents had poor knowledge about malaria signs and symptoms [37].

In the present study, low level of awareness was observed with only $50(33.6 \%)$ and $26(17.4 \%)$ participants correctly identified under five children and pregnant women as the most susceptible groups for malaria infection, respectively. However, a high level of awareness was reported in other studies in Ethiopia where $85.1-90.3 \%$ and $59.0 \%-62.3 \%$ of respondents were aware that under 5 children and pregnant mothers are the most risk groups, respectively $[25,30]$.

With regard to knowledge in intervention measures for indoor prevention and vector control, 120 (81.9\%) participants understood ITN as malaria preventive method. It was higher than reports from Myanmar that $65.6 \%$ of respondents mentioned sleeping with bed net as malaria preventive method [38]. However, it was lower when compared with two previous studies from Ethiopia with $95.4 \%$ [28] and 97.55\% [39] of respondents agreed on the fact that use of ITN can prevent from malaria.

This study shows that $130(87.6 \%)$ respondents agreed to seriousness and threat posed by malaria. It was in line with results from Raya Azebo district (90.8\%) [24]. On the other hand, it was higher than the study findings in Tigray region (65.9\%) [27]. One hundred twenty-three (82.6\%) participants agreed with the statement that malaria is a preventable disease, which was comparable with the study conducted in Raya Azebo district where $87.9 \%$ respondents believed that malaria is a preventable disease [24]. However, it was lower than results from Shewa Robit where $90.58 \%$ respondents believed that malaria is a preventable disease [20]. On the contrary, it was higher than the study done in Tigray region (74.7\%) [27] and Paksong district of Lao PDR (78\%) [34].

A total of 44 (29.5\%), 50 (33.6\%), and 55 (36.9\%) participants had good, satisfactory, and poor knowledge about malaria, respectively. This is lower when compared with a study from Southern Ethiopia, where $74.3 \%$ of respondents had good knowledge while the remaining $25.7 \%$ had poor knowledge [20]. It was also lower than the study conducted in Champasack Province of Lao PDR where $59.1 \%$ of respondents had good knowledge [40]. On the other hand, the knowledge level in the present study was higher as compared to previous findings from Mumbai. In Mumbai, $39.7 \%, 53.7 \%$, and $5.8 \%$ had low, average, and high level of knowledge, respectively [41]. Differences in the overall 
civilization, health education and implementation coverage, health policy, and sociocultural habits are proposed factors for variations in knowledge level about malaria.

Statistically significant associations were observed on knowledge with age group, educational level, family size, attitude level, and practice level. Participants of older age group, i.e., $\geq 36$, possessed significantly high percentage of good knowledge $(47.7 \%)$ as compared to other age groups, 15-25 (27.3\%) and 26-35 (25.0\%). Likewise, respondents who can read and write and above had significantly high frequency of good knowledge (59.1\%) than those who were unable to read and write $(40.9 \%)$. The participants whose family size was $\geq 5$ had higher proportion of good knowledge $(65.9 \%)$ as compared to family members were $<5$ (34.1\%). Moreover, the respondents who had positive attitude have high percentage of good knowledge (93.2\%) than who had negative attitude towards malaria (6.8\%). Finally, those who had poor practice had higher frequency of good knowledge $(63.6 \%)$ than those who had poor practice level (36.4\%).

This study revealed that $115(77.2 \%)$ participants had positive attitude while $34(22.8 \%)$ had negative attitude towards malaria in terms of its seriousness or threat, prevention and control. This is lower when compared with studies in Karachi where $97 \%$ of the respondents had good attitude [36]. To the contrary, it was higher than the study from Amhara National Regional State of Ethiopia with 69\% respondents having positive attitude towards malaria [35]. Moreover, it was higher than the study conducted in Mumbai, that $16.9 \%$ of respondents had positive attitude [41]. In the present study, participants who had $<5$ family size were 6.8 times more positive attitude compared to those with $\geq 5$ family size.

Result observed in this study revealed that 52 (34.9\%) participants had good and $97(65.1 \%)$ had poor practice towards malaria prevention and control measures. This is lower when compared to studies in Southern Ethiopia [9], Hadya zone [12], and Karachi [36] where 67.7\%, 32.3\%, and $59 \%$ of the study participants had good practice, respectively. Conversely, studies from LAO PDR reported that only $5.7 \%$ had good practice [40]. Variations in knowledge about malaria, availability of intervention tools, attention given by health authorities, and local transmission level of the disease might bring differences in the attitude and practice levels about malaria. Male had around 52\% less good practice as compared to female. The limitation of this study is that we used nonvalidated questionnaire adapted from recent similar studies [9-12, 20, 25, 27] and translated to Amharic language. We recommend implementation of community health education focusing on malaria transmission, health and economic outcome, intervention approaches, and how people can involve in intervention activities in the local context.

\section{Conclusions}

The current study illustrated that prevalence of malaria among febrile patients in the study area was high with relative dominance of $P$. vivax. It has also been indicated that study participants had encouraging attitudes. Nonetheless, inadequate knowledge about malaria transmission and prevention as well as poor practice towards malaria control and prevention was noticed in the present study.

\section{Abbreviations \\ IRS: Indoor residual spraying \\ ITNs: Long-lasting insecticide-treated nets \\ KAP: Knowledge, attitude, and practice \\ RDT: Rapid diagnostic tests.}

\section{Data Availability}

The authors confirm that all data underlying the findings are fully available without restriction. All relevant data are within the manuscript.

\section{Ethical Approval}

The research was carried out after ethical approval was obtained from the Institutional Review Committee of College of Medicine and Health Sciences, Bahir Dar University. Additionally, supportive letters were obtained from Amhara Public Health Institute, West Gojjam Zone Health Department, and Bahir Dar Zuria district health office and permission was obtained from each health center authorities.

\section{Consent}

Oral informed consent was obtained from voluntary participants and parents or guardians for children during data collection. Consent for publication is not necessary.

\section{Disclosure}

The funding body did not have a role in the design of the study and collection, analysis, and interpretation of data and in writing the manuscript.

\section{Conflicts of Interest}

The authors declare that they have no conflicts of interest.

\section{Authors' Contributions}

ZD and GA involved in proposal writing, designed the study, and participated in all implementation stages of the project. ZD and GA also analyzed the data and finalized the write up of the manuscript. MY, AG, and BT were responsible for critically revising the proposal and the manuscript. All authors reviewed and approved the final manuscript.

\section{Acknowledgments}

We thank Bahir Dar University for partially funding the project. We also thank West Gojjam Zonal Health Department, Bahir Dar Zuria District Health Office, and health centers in Bahir Dar Zuria District for their permission 
and cooperation at the time of data collection. Finally, we also thank those who participated in the study. This study was partially funded by the Bahir Dar University.

\section{References}

[1] WHO, World Malaria Report, World Health Organization Geneva, 2017, Nov. 2019, http://www.gbchealth.org/wpcontent/uploads/2019/05/World-Malaria-Report-2018.pdf.

[2] WHO, World Malaria Report, World Health Organization Geneva, 2019, May 2020, https://creativecommons.org/ licenses/by-nc-sa/3.0/igo.

[3] A. Alelign and T. Dejene, "Current status of malaria in Ethiopia: evaluation of the burden, factors for transmission and prevention methods," Acta Parasitologica Globalis, vol. 7, no. 1, pp. 01-06, 2016.

[4] Ethiopian Public Health Institute Federal Ministry of Health, Malaria Laboratory Diagnosis External Quality Assessment Scheme Guidelines, Addis Ababa, 2017.

[5] Federal Ministry of Health, National Malaria Guidelines, Addis Ababa, 2017.

[6] L. Garcia, Diagnostic Medical Parasitology, American society for microboilogy Press:142-160, Washington, D.C., 5th ed. edition, 2007.

[7] CDC, Malaria about malaria disease, 2019, Nov. 2019, https:// www.cdc.gov/malaria/about/disease.html.

[8] Federal Ministry of Health, Guideline for Malaria Epidemic Prevention and Control in Ethiopia, Federal Ministry of Health, Ethiopia, Addis Ababa, 2nd ed. edition, 2004.

[9] Y. Legesse, A. Tegegn, T. Belachew, and K. Tushune, "Knowledge, attitude and practice about malaria transmission and its preventive measures among households in urban areas of Assosa zone, Western Ethiopia," Ethiopian Journal of Health Development, vol. 21, no. 2, pp. 157-165, 2007.

[10] N. M. Shimaponda-Mataa, E. Tembo-Mwase, M. Gebreslasie, and S. Mukaratirwa, "Knowledge, attitudes and practices in the control and prevention of malaria in four endemic provinces of Zambia. Southern African," Journal of Infectious Diseases, vol. 32, no. 1, pp. 29-39, 2017.

[11] H. D. Mazigo, E. Obasy, W. Mauka et al., "Knowledge, attitudes, and practices about malaria and its control in rural Northwest Tanzania," Malaria Research and Treatment, vol. 2010, 9 pages, 2010.

[12] T. G. Fuge, S. Y. Ayanto, and F. L. Gurmamo, "Assessment of knowledge, attitude and practice about malaria and ITNs utilization among pregnant women in Shashogo District, southern Ethiopia," Malaria Journal, vol. 14, no. 1, p. 235, 2015.

[13] Bureau of Finance and Economic Development of Amhara (BoFED), Support to Community-Led Accelerated WASH in Ethiopia Revised Project Document for COWASH Phase I, 6/2011-6/2013 Phase II, 7/2013-6/2016, FDRE and MoFED, 2011.

[14] President's Malaria Initiative (PMI), Ethiopia Malaria Operational Plan, Addis Ababa, Ethiopia, 2017.

[15] D. G. Feleke, S. Tarko, and H. Hadush, "Performance comparison of CareStart ${ }^{\mathrm{TM}} \mathrm{HRP} 2 / \mathrm{pLDH}$ combo rapid malaria test with light microscopy in north-western Tigray, Ethiopia: a cross-sectional study," BioMedicalCenter Infectious Diseases, vol. 17, no. 1, p. 399, 2017.

[16] B. S. Bloom, "Taxonomy of educational objectives," Cognitive Domain. New York: McKay, vol. 20, no. 1, p. 24, 1956.
[17] R. Likert, "A technique for the measurement of attitudes," Archives of Psychology, vol. 22, 1932.

[18] T. F. Belaynesh, Knowledge, Attitude and Practice towards Malaria among People Attending in Mekaneeyesus Primary Hospital, A cross-sectional study, South Gondar, Northwestern Ethiopia, 2020.

[19] E. Molla and B. Ayele, "Prevalence of malaria and associated factors in Dilla town and the surrounding rural areas, Gedeo Zone, Southern Ethiopia," Journal of Bacteriology \& Parasitology, vol. 6, no. 5, p. 1, 2015.

[20] A. Abate, A. Degarege, and B. Erko, "Community knowledge, attitude and practice about malaria in a low endemic setting of Shewa Robit Town, northeastern Ethiopia," BMC Public Health, vol. 13, no. 1, p. 312, 2013.

[21] B. Moges, B. Amare, Y. Belyhun et al., "Comparison of CareS$\operatorname{tart}^{\mathrm{TM}}$ HRP2/pLDH COMBO rapid malaria test with light microscopy in north-west Ethiopia," Malaria Journal, vol. 11, no. 1, p. 234, 2012.

[22] F. Tadesse, A. W. Fogarty, and W. Deressa, "Prevalence and associated risk factors of malaria among adults in East Shewa Zone of Oromia Regional State, Ethiopia: a cross-sectional study," BMC Public Health, vol. 18, no. 1, p. 25, 2018.

[23] K. T. Bidu and Z. K. Babure, "Prevalence of malaria and associated factors among febrile patients visiting Kalala Health Center in Haro Limmu Woreda, East Wollega Zone, Western Ethiopia, 2016," Epidemiology (Sunnyvale), vol. 9, no. 365, 2019.

[24] K. Tesfay, M. Yohannes, F. Mardu, B. Berhe, and H. Negash, "Assessment of community knowledge, practice, and determinants of malaria case households in the rural area of Raya Azebo district, Northern Ethiopia, 2017," PLoS One, vol. 14, no. 10, article e0222427, 2019.

[25] A. Henok, "Knowledge and practice towards prevention and control of malaria in Tepi Town, Sheka zone, Southwestern Ethiopia," Health Science Journal, vol. 10, p. 1, 2015.

[26] T. Gobena, Y. Berhane, and A. Worku, "Women's knowledge and perceptions of malaria and use of malaria vector control interventions in Kersa, Eastern Ethiopia," Global Health Action, vol. 6, no. 1, p. 20461, 2013.

[27] J. Paulander, H. Olsson, H. Lemma, A. Getachew, and S. M. San, "Knowledge, attitudes and practice about malaria in rural Tigray, Ethiopia," Global Health Action, vol. 2, no. 1, p. 1839, 2009.

[28] T. A. Hamza, N. N. Azmach, and A. A. Husen, "Community knowledge, attitude and practice about malaria and mosquito biting behavior in Southern Ethiopia," American Journal of BioScience, vol. 5, no. 5, pp. 80-88, 2017.

[29] W. Deressa, A. Ali, and D. Hailemariam, "Malaria-related health-seeking behaviour and challenges for care providers in rural Ethiopia: implications for control," Journal of Biosocial Science, vol. 40, no. 1, pp. 115-135, 2008.

[30] A. Astatkie, "Knowledge and practice of malaria prevention methods among residents of Arba Minch Town and Arba Minch Zuria District, Southern Ethiopia," Ethiopian Journal of Health Sciences, vol. 20, no. 3, 2011.

[31] R. Singh, J. Musa, S. Singh, and U. V. Ebere, "Knowledge, attitude and practices on malaria among the rural communities in Aliero, Northern Nigeria," Journal of Family Medicine and Primary Care, vol. 3, no. 1, pp. 39-44, 2014.

[32] D. Z. Munisi, A. A. Nyundo, and B. C. Mpondo, "Knowledge, attitude and practice towards malaria among symptomatic 
patients attending Tumbi referral hospital: a cross-sectional study," PLoS One, vol. 14, no. 8, article e220501, 2019.

[33] M. Soleimani-Ahmadi, H. Vatandoost, M. Zare, A. Alizadeh, and M. Salehi, "Community knowledge and practices regarding malaria and long-lasting insecticidal nets during malaria elimination programme in an endemic area in Iran," Malaria Journal, vol. 13, no. 1, pp. 1-7, 2014.

[34] K. W. Hlongwana, M. L. Mabaso, S. Kunene, D. Govender, and R. Maharaj, "Community knowledge, attitudes and practices (KAP) on malaria in Swaziland: a country earmarked for malaria elimination," Malaria Journal, vol. 8, no. 1, p. 29, 2009.

[35] Z. Aderaw and M. Gedefaw, "Knowledge, attitude and practice of the community towards malaria prevention and control options in anti-malaria association intervention zones of Amahara National Regional State, Ethiopia," Journal of Tropical Diseases, vol. 1, no. 118, 2013.

[36] A. Bilal, W. A. Siddiqui, and F. A. Mansuri, "Knowledge, attitude and practice (KAP) about malaria among inhabitants of a slum area of Karachi," Annals of Abbasi Shaheed Hospital and Karachi Medical and Dental College, vol. 18, pp. 79-85, 2013.

[37] G. B. Serengbe, J. M. Moyen, R. Fioboy et al., "Knowledge and perceptions about malaria in communities in four districts of the Central African Republic," BMC Research Notes, vol. 8, no. 1, pp. 1-6, 2015.

[38] Z. Soe and W. H. Shwe, "Knowledge, attitude and practice on insecticide treated nets in Myanmar," International Journal of Collaborative Research on Internal Medicine \& Public Health, vol. 5, no. 6, 2013.

[39] Z. Tomass, T. Dejene, and D. Kidane, "Knowledge, attitude and practice (KAP) about insecticide treated net (ITN) usage against malaria in Kolla Tembien district, Tigray, Ethiopia," Momona Ethiopian Journal of Science, vol. 3, no. 2, 2011.

[40] C. Thanabouasy, T. Pumpaibool, and N. Kanchanakhan, "Assessment of knowledge, attitude and practice regarding malaria prevention towards population in Paksong District Champasack Province, Lao PDR," Journal of Health Research, vol. 23, pp. 11-15, 2009.

[41] S. Ambadas, S. Kadam, P. Desai, and D. Deogune, "Knowledge, attitude and practices of people towards malaria in squatter's colony, Malvani, Malad, Mumbai," International Journal of Scientic Research, vol. 4, no. 5, pp. 2277-8179, 2015. 